TRANSACTIONS OF THE

AMERICAN MATHEMATICAL SOCIETY

Volume 362, Number 5, May 2010, Pages 2415-2434

S 0002-9947(09)05030-2

Article electronically published on December 17, 2009

\title{
ACTIONS OF SEMISIMPLE LIE GROUPS PRESERVING A DEGENERATE RIEMANNIAN METRIC
}

\author{
E. BEKKARA, C. FRANCES, AND A. ZEGHIB
}

\begin{abstract}
We prove a rigidity of the lightcone in Minkowski space. It is (essentially) the unique space endowed with a lightlike metric and supporting an isometric nonproper action of a semisimple Lie group.
\end{abstract}

\section{INTRODUCTION}

Our subject of study here is lightlike metrics on smooth manifolds. First, a lightlike scalar product on a vector space $E$ is a symmetric bilinear form $b$ which is positive but nondefinite and which has exactly a 1-dimensional kernel. If $E$ has dimension $1+n$, then in some linear coordinates $\left(x^{0}, x^{1}, \ldots, x^{n}\right)$, the associated quadratic form $q$ can be written $q=\left(x^{1}\right)^{2}+\ldots+\left(x^{n}\right)^{2}$. A lightlike metric $h$ on a manifold $M$ is a smooth tensor which is a lightlike scalar product on the tangent space of each point.

1.0.1. Characteristic foliation. The kernel of $h$ is a 1-dimensional sub-bundle $N \subset$ $T M$ and thus determines a 1-dimensional foliation $\mathcal{N}$ called the characteristic (or null, normal, radical, isotropic,...) foliation of $h$. By definition any null curve (i.e. a curve with everywhere isotropic speed) of $(M, h)$ through $x$ is contained in the null leaf $\mathcal{N}_{x}$. The abstract normal bundle of $\mathcal{N}$, i.e. the quotient $T M / N$, is a Riemannian vector bundle. Conversely, a lightlike metric is the data of a 1-dimensional foliation together with a Riemannian metric on its normal bundle.

1.1. Major motivations. Lightlike geometry appears naturally in many geometric situations. We now list some natural examples motivating their study.

1.1.1. Submanifolds of Lorentz manifolds. Let $M$ be a submanifold in a Lorentz manifold $(V, g)$. The metric $g$ is nondegenerate with signature $-+\ldots+$. However, for a given $x \in M$, the restriction $h_{x}$ of $g$ to $T_{x} M$ does not necessarily have the same signature. Two easy stable situations are those where $h_{x}$ is everywhere of Riemannian type ( $M$ is spacelike) or $h_{x}$ is everywhere of Lorentzian type ( $M$ is timelike). In both cases, all the submanifold theory valid in the Riemannian context generalizes: there is a well-defined shape operator which satisfies the Gauss and Codazzi equations.

Received by the editors November 26, 2007.

2010 Mathematics Subject Classification. Primary 53B30, 53C22, 53C50.

Key words and phrases. Lightlike metric, lightcone, isotropic direction.

The first author was partially supported by the project CMEP 05 MDU $641 \mathrm{~B}$ of the Tassili program.

(C)2009 American Mathematical Society Reverts to public domain 28 years from publication 
The delicate situation occurs when $h_{x}$ is degenerate for any $x$. Because the ambient metric has Lorentz signature, $h_{x}$ is then lightlike as defined above. Unfortunately, by opposition to the previous cases, these lightlike submanifolds are generally "too poor" to generate a coherent extrinsic local metric differential geometry. Let us give examples of interesting lightlike submanifolds 11

- Horizons of domains of dependence and black holes. Unfortunately, they have an essential lack: their lower smoothness. Actually, one can believe that smooth horizons are sufficiently rigid to be classifiable (see for instance 22] for a quick definition of domains of dependence and 6, 20, 13, for more details, examples and rigidity).

- Characteristic hypersurfaces of the wave equation. On a Lorentz manifold, a wave operator defines a distinguished class of hypersurfaces called characteristic 2 There is a nice interpretation of lightlike hypersurfaces in terms of propagation of waves: a hypersurface is degenerate exactly if it is characteristic for the wave equation on the ambient Lorentz space (see [12]). However, except for some general elementary facts, no systematic study of the extrinsic geometry of such hypersurfaces seems to be available in the literature. One of these known facts is that their null curves, called bi-characteristics of the wave equation, are unparametrized geodesics in the ambient space (this is not true for lightlike submanifolds of higher codimension). Another property is that being a lightlike submanifold is conformally invariant (in particular, unparametrized null geodesics in Lorentz manifolds are conformally invariant!).

- Lightlike geodesic hypersurfaces. They are characterized by the fact that their lightlike metrics are basic (see the example in 1.2 .1 ). They inherit a connection from the ambient space. See [8, 9, 23, 24] for their use in Lorentz dynamics.

- Degenerate orbits of Lorentz isometric actions. Let $G$ be a Lie group acting isometrically on a Lorentz manifold $(V, g)$. Then any orbit which is lightlike at a point is lightlike everywhere and hence yields an embedded lightlike submanifold in $V$. The problem of understanding these lightlike orbits, and more generally degenerate invariant submanifolds, is essential when studying such isometric actions.

- Terminology. We believe that the choice of the word "lightlike" is justified by the relationship between lightlike submanifolds and fields on the one hand, and geometrical as well as physical optics in general Relativity on the other hand (see for instance [21). Although this terminology seems natural here, it is less adapted to the general situation of "singular pseudo-Riemannian" metrics (compare with [10, 17]).

1.1.2. From submanifolds to intrinsic lightlike geometry. In the last example given above, when we restrict the action of the Lie group $G$ to a lightlike orbit, we are led to study the isometric action of $G$ on a lightlike submanifold in a Lorentzian manifold. The submanifold structure is actually irrelevant for this problem, and

\footnotetext{
1 The first two examples come from mathematical Relativity. We cannot recall detailed definitions, but in a few words, a Cauchy hypersurface in a Lorentz manifold is a spacelike hypersurface that captures all the causal structure: every timelike curve can be extended to meet it exactly once. For any spacelike hypersurface, its domain of dependence is the maximal open set in which it is a Cauchy hypersurface. The horizon of the hypersurface is the boundary of its domain of dependence.

2 In order to solve an initial data problem for the wave equation, the data must be given on a Cauchy hypersurface. The completely opposite situation occurs when the hypersurface is characteristic.
} 
the pertinent framework is that of isometric actions on abstract lightlike manifolds. The main difficulty when dealing with this intrinsic formulation is that we lose the rigidity of the ambient action since, as we will see below, the isometry group of a lightlike manifold can be infinite-dimensional.

1.2. Two fundamental examples. We now give two important examples of lightlike geometries, which are in some sense antagonistic.

1.2.1. The most flexible example: transversally Riemannian flows. The linear situation reduces to the case of $\mathbb{R}^{0, n}$, i.e. $\mathbb{R}^{1+n}$ with coordinates $\left(x^{0}, x^{1}, \ldots, x^{n}\right)$ endowed with the lightlike quadratic form $q=\left(x^{1}\right)^{2}+\ldots+\left(x^{n}\right)^{2}$.

We will denote its linear orthogonal group by $O(0, n)$ (this is somehow natural since it is reminiscent of the notation $O(1, n))$. We have:

$$
O(0, n)=\left\{\left(\begin{array}{cccc}
\lambda & a_{1} & \ldots & a_{n} \\
0 & & & \\
\cdot & & A & \\
\cdot & & &
\end{array}\right) \in G L(1+n, \mathbb{R}), A \in O(n), \lambda, a_{i} \in \mathbb{R}\right\}
$$

It is naturally isomorphic to the affine similarity group $\mathbb{R} \times E u c_{n}=\mathbb{R} . O(n) \ltimes \mathbb{R}^{n}$ (where $E u c_{n}=O(n) \ltimes \mathbb{R}^{n}$ denotes the group of rigid motions of the Euclidean space of dimension $n$ ).

Let us now see $\mathbb{R}^{1+n}$ as a lightlike manifold. The group of its affine isometric transformations is $O(0, n) \ltimes \mathbb{R}^{1+n}$. Contrary to the nondegenerate case, there is here a huge, infinite-dimensional group of nonaffine isometries. For example, any

$$
\psi:\left(x^{0}, x^{1}, \ldots, x^{n}\right) \mapsto\left(\psi_{1}\left(x^{0}, x^{1}, \ldots, x^{n}\right), \psi_{2}\left(x^{1}, \ldots, x^{n}\right)\right),
$$

where $\psi_{2} \in E u c_{n}$, and $\psi_{1}: \mathbb{R}^{n+1} \rightarrow \mathbb{R}$ is a smooth function with $\frac{\partial \psi_{1}}{\partial x^{0}} \neq 0$, is an isometry.

- More generally, let us consider $(L, g)$ as a Riemannian manifold and $M=\mathbb{R} \times L$ endowed with the lightlike metric $0 \oplus g$. The null foliation is given by the $\mathbb{R}$-factor, and the metric does not depend on the coordinate along it. Here we also get an infinite-dimensional group of isometric transformations given by $\psi:(t, l) \in$ $\mathbb{R} \times L \mapsto\left(\psi_{1}(t, l), \psi_{2}(l)\right)$, where $\psi_{2}$ is an isometry of $L$, e.g. $\psi_{2}$ is the identity map, and $\frac{\partial \psi_{1}}{\partial t} \neq 0$.

Conversely, assume that the lightlike metric $(M, h)$ is such that there exists a nonsingular vector field $X$ tangent to the characteristic foliation and satisfying $L_{X} h=0$. Then locally, there is a metric splitting $M=\mathbb{R} \times L$ as above. Observe that any vector field collinear to $X$ will actually preserve $h$. In other words, any vector field orienting the characteristic foliation $\mathcal{N}$ preserves $h$. We call the lightlike metric basic in this case. This terminology is justified by the fact that $h$ is the pull-back by the projection map $M \rightarrow L$ of the Riemannian metric on the basis $L$.

- A 1-dimensional foliation $\mathcal{N}$ on a manifold $M$ is transversally Riemannian (one then says $\mathcal{N}$ is a transversally Riemannian flow) if it is the characteristic foliation of some lightlike metric $h$ on $M$, this metric being moreover preserved by the local vector fields tangent to $\mathcal{N}$. This data is strictly equivalent to that of a basic lightlike metric on $M$. Of course, the usual classical definition does not involve lightlike metrics. The reader will find in [7, 19] an introduction to the theory of transversally Riemannian foliations, with sharp conclusions in the 1-dimensional case. 
The isometry group of a basic lightlike metric contains at least all flows tangent to $\mathcal{N}$, so that it is infinite-dimensional and surely not so beautiful. However, these metrics are somehow tame, since at least locally the metric is encoded in the associated Riemannian one. Moreover, it was proved by D. Kupeli [17] (and reproduced in many other places) that some kind of Levi-Civita connection exists exactly if the lightlike metric is basic. The connection is never unique, so it is often necessary to consider additional structures. The most useful one is that of screen, mostly developed in [10, which allows us to develop "calculus" and sometimes get invariant quantities (see for instance [3]). Nevertheless, there is generally no distinguished screen left invariant by the isometry group, so this notion will not be helpful to us.

1.2.2. The example of the lightcone in Minkowski space. We will now consider an opposite situation where the isometry group is "big", although remaining finitedimensional. Let $\operatorname{Min}_{1, n}$ be the Minkowski space of dimension $1+n$, i.e. $\mathbb{R}^{1+n}$ endowed with the form $q=-x_{0}^{2}+x_{1}^{2}+\ldots+x_{n}^{2}$. The isotropic positive lightcone $C o^{n}$ is the set $\left\{q(x)=0, x_{0}>0\right\}$. The metric induced by $q$ on $C o^{n}$ is lightlike. The subgroup $O^{+}(1, n) \subset O(1, n)$ preserving the cone $C o^{n}$ acts isometrically on it. This action is in fact transitive so that $C o^{n}=O^{+}(1, n) / E u c_{n-1}$ becomes a lightlike homogeneous space with isotropy group $E u c_{n-1}=O(n-1) \ltimes \mathbb{R}^{n-1}$, the group of rigid motions of the Euclidean space of dimension $n-1$.

A key observation is:

Theorem 1.1 (Liouville Theorem for lightlike geometry). For $n \geq 3$, any isometry of $\mathrm{Co}^{n}$ belongs to $\mathrm{O}^{+}(1, n)$. This is true even locally for $n \geq 4$ : any isometry between two connected open subsets of $\mathrm{Co}^{n}$ is the restriction of an element of $O^{+}(1, n)$.

- For $n=3$, the group of local isometries is in one-to-one correspondence with the group of local conformal transformations of $\mathbf{S}^{2}$.

- For $n=2$, there is no rigidity at all, even globally, since to any diffeomophism of the circle corresponds an isometry of $\mathrm{Co}^{2}$.

This theorem, which will be proved in $₫ 2$ shows in particular that for $n \geq 3$, $C o^{n}$ is a homogeneous lightlike manifold with isometry group $O^{+}(1, n)$. We remark that for the sake of simplicity we will often use the notation $O(1, n)$ for any finite index subgroup of $O(1, n)$. Actually, to be precise, we can say that our geometric descriptions of objects are always given up to a finite cover.

It seems likely that being homogeneous and having a maximal isotropy $O(0, n-1)$ characterizes the flat case, i.e. $\mathbb{R}^{0, n-1}$, and having a maximal unimodular isotropy, i.e. $E u c_{n-1}$, characterizes the lightcone. In some sense the lightcone is the maximal symmetric nonflat lightlike space, analogous to spaces of constant nonzero curvature in the pseudo-Riemannian case.

1.3. Statement of results. The present article contains detailed proofs of the results announced in [5]. Before giving the statements, let us recall that two lightlike metrics $h$ and $h^{\prime}$ on a manifold $M$ are said to be homothetic if $h=\lambda h^{\prime}$ for some real $\lambda>0$. A Lie group acts locally faithfully on $M$ if the kernel of the action is a discrete subgroup.

One motivation of the present work was Theorem 1.6 of [9], which we state here as follows. 
Theorem $1.2(9])$. Let $G$ be a connected group with finite center, locally isomorphic to $O(1, n)$ or $O(2, n), n \geq 3$. If $G$ acts isometrically on a Lorentz manifold and has a degenerate orbit with noncompact stabilizer, then $G$ is locally isomorphic to $O(1, n)$ and the orbit is homothetic to the lightcone $C o^{n}$.

Here, we prove an intrinsic version of this result:

Theorem 1.3. Let $G$ be a noncompact semisimple Lie group with finite center acting locally faithfully, isometrically and nonproperly on a lightlike manifold $(M, h)$. Assume that $G$ has no factor locally isomorphic to $S L(2, \mathbb{R})$. Then, looking if necessary at a finite cover of $G$ :

- $G=H \times H^{\prime}$, where $H$ is locally isomorphic to $O(1, n)$.

- $G$ has an orbit which is homothetic, up to a finite cover, to a metric product $C o^{n} \times N$, where $N$ is a Riemannian $H^{\prime}$-homogeneous manifold. The action of $H \times H^{\prime}$ on $\mathrm{Co}^{n} \times N$ is the product action.

Using this theorem and working a little bit more, we can also handle the case where some factors of $G$ are locally isomorphic to $S L(2, \mathbb{R})$ when the action is transitive. The following result can be thought of as a converse to Theorem 1.1;

Corollary 1.4. Let $G$ be a noncompact semisimple Lie group with finite center, acting locally faithfully, isometrically, transitively and nonproperly on a lightlike manifold $(M, h)$, i.e. $M$ is a homogeneous lightlike space $G / I$, with a noncompact isotropy group I. Then a finite cover of $G$ is isomorphic to $O(1, n) \times H^{\prime}$, where $n \geq 2$ and $H^{\prime}$ is semisimple.

- If $n \neq 2$, then up to finite cover, the manifold $M$ is homothetic to a metric product $C o^{n} \times N$, where $N$ is an $H^{\prime}$-homogeneous Riemannian space.

- If $n=2$, then up to finite cover, $M$ is either homothetic to a metric product $C o^{1} \times N$ as above or is a topological product $C o^{2} \times N$, and there is a finite-dimensional family of G-homogeneous lightlike metrics, which is, up to homothety, in natural one-to-one correspondence with the linear forms on $\mathbb{R}^{\operatorname{dim} N}$. Up to homothety, any such $h$ induces the standard lightlike metric on $\mathrm{Co}^{2}$ and an $\mathrm{H}^{\prime}$-homogeneous Riemannian metric on $N$.

In any case, the action of $G$ on $M$ is the product action.

The nonproperness assumption is essential in the previous theorems. If one removes it, "everything becomes possible". Indeed, consider a Lie group $L$ and a lightlike scalar product on its Lie algebra $\mathfrak{l}$. Translating it on $L$ by left multiplication yields a lightlike metric for which the left action of $L$ is isometric.

It is quite surprising that these kinds of global rigidity theorems can be proved in the framework of lightlike metrics, which are not rigid geometric structures (see 1.2.1). Here, it is in some sense the algebraic assumption of semisimplicity which makes the situation rigid. However, since any Lie algebra is a semidirect product of a semisimple and a solvable one, it is natural to first consider actions of semisimple Lie groups.

When the manifold $M$ is compact, only one simple Lie group can act isometrically, as shows:

Theorem 1.5. Let $G$ be a noncompact simple Lie group with finite center acting isometrically on a compact lightlike manifold $(M, h)$. Then $G$ is a finite covering of $\operatorname{PSL}(2, \mathbb{R})$, and all the orbits of $G$ are closed, 1-dimensional, and lightlike. 
1.4. The mixed signature case: sub-Lorentz metrics. This notion will naturally model the situation of general submanifolds in Lorentz manifolds. A subLorentz metric $g$ on $M$ is a symmetric covariant 2 -tensor which is at each point a scalar product of either Lorentz, Euclidean, or lightlike type. The point is that we allow the type to vary over $M$. So if $(L, h)$ is a Lorentz manifold and $M$ is a submanifold of $L$, then the restriction on $h$ to $M$ is a sub-Lorentz metric (this fact raises the inverse problem, i.e. the isometric embedding of sub-Lorentz metrics in Lorentz manifolds). We think it is worthwhile to investigate the geometry of these natural and rich structures (see for instance [18, for a research of normal forms of these metrics in dimension 2).

Here we restrict our investigation to an adaptation of our lightlike results to this sub-Lorentz situation.

1.4.1. Lorentz dynamics. Recall the three fundamental examples of Lorentz manifolds having an isometry group which acts nonproperly. They are just the universal spaces of constant curvature:

(1) The Minkowski space: $\operatorname{Min}_{1, n-1}=O(1, n-1) \ltimes \mathbb{R}^{n} / O(1, n-1)$.

(2) The de Sitter space $d S_{n}=O(1, n) / O(1, n-1)$.

(3) The anti-de Sitter space $A d S_{n}=O(2, n-1) / O(1, n-1)$.

In the case of Minkowski space, the isometry group is not semisimple.

The Lorentz and lightlike dynamics are unified in the following statement, which is basically a corollary of Theorem 1.3 and results of [4]:

Theorem 1.6. Let $G$ be a semisimple group with finite center, no compact factor and no local factor isomorphic to $S L(2, \mathbb{R})$, acting isometrically nonproperly on a sub-Lorentz manifold $M$. Then up to a finite cover, $G$ has a factor $G^{\prime}$, isomorphic to $O(1, n)$ or $O(2, n)$ and having some orbit homothetic to $d S_{n}, A d S_{n}$ or $C o^{n}$.

\section{Preliminaries}

2.1. Proof of Theorem 1.1. The metric on $C o^{n}$ is just the metric $0 \oplus e^{2 t} g_{\mathbf{S}^{n-1}}$ on $\mathbb{R} \times \mathbf{S}^{n-1}$. An isometry $f$ of $C o^{n}$ is of the form $(t, x) \mapsto(\lambda(t, x), \phi(x))$. A simple calculation proves that $f$ is isometric iff at any point $(t, x) \in \mathbb{R} \times \mathbf{S}^{n-1}$,

$$
\phi^{*} g_{\mathbf{S}^{n-1}}=e^{2(t-\lambda(t, x))} g_{\mathbf{S}^{n-1}} .
$$

So any local isometry of $C o^{n}$ is of the form $(t, x) \mapsto(t-\mu(x), \phi(x))$, with $\phi$ a local conformal transformation of the sphere satisfying $\phi^{*} g_{\mathbf{S}^{n-1}}=e^{2 \mu} g_{\mathbf{S}^{n-1}}$. Thus, the different rigidity phenomena are just consequences of classical analogous rigidity results for conformal transformations on the sphere.

2.2. $S L(2, \mathbb{R})$-homogeneous spaces. Understanding these spaces is worthwhile in our context since one can take advantage of restricting the $G$-action to small simpler groups, e.g. $S L(2, \mathbb{R})$ or a finite cover of it, which always exist in semisimple Lie groups.

2.2.1. Notation. Let $S L(2, \mathbb{R})$ be the Lie group of $2 \times 2$-matrices with determinant 1. It is known that any 1-parameter subgroup of $S L(2, \mathbb{R})$ is conjugate to one of 
the following:

$$
\begin{gathered}
A^{+}=\left\{\left(\begin{array}{cc}
e^{t} & 0 \\
0 & e^{-t}
\end{array}\right), t \in \mathbb{R}\right\}, N=\left\{\left(\begin{array}{ll}
1 & t \\
0 & 1
\end{array}\right), t \in \mathbb{R}\right\} \\
\text { or } K^{+}=\left\{\left(\begin{array}{cc}
\sin t & -\cos t \\
\cos t & \sin t
\end{array}\right), t \in \mathbb{R}\right\} .
\end{gathered}
$$

The corresponding derivatives of $A^{+}$and $N$ at the identity are

$$
X=\left(\begin{array}{cc}
1 & 0 \\
0 & -1
\end{array}\right) \text { and } Y=\left(\begin{array}{ll}
0 & 1 \\
0 & 0
\end{array}\right) .
$$

Together with $Z=\left(\begin{array}{ll}0 & 0 \\ 1 & 0\end{array}\right), X$ and $Y$ span the Lie algebra $\mathfrak{s l}(2, \mathbb{R})$ and satisfy the bracket relations

$$
[X, Y]=2 Y, \quad[X, Z]=-2 Z \text { and } \quad[Y, Z]=X .
$$

As usual, we denote by $A$ (resp. $K$ ) the subgroup generated by $A^{+},-A^{+}$(resp. $\left.K^{+},-K^{+}\right)$.

Let $\operatorname{Aff}(\mathbb{R})$ be the subgroup of upper triangular matrices,

$$
\operatorname{Aff}(\mathbb{R})=A . N=\left\{\left(\begin{array}{cc}
a & b \\
0 & a^{-1}
\end{array}\right) \in S L(2, \mathbb{R})\right\}
$$

and $\mathfrak{a f f}(\mathbb{R})$ be its Lie algebra.

Disconnected 1-dimensional subgroups of $\operatorname{Aff}(\mathbb{R})$ can be constructed as follows. Let $\Gamma_{0}$ be a cyclic subgroup of $A$ generated by an element $\gamma \in A$. The semidirect product $\Gamma_{0} \ltimes N$ is then a closed, 1-dimensional, disconnected subgroup of $\operatorname{Aff}(\mathbb{R})$. Conversely, any closed 1-dimensional disconnected subgroup of $A f f(\mathbb{R})$ is obtained in this manner.

Thanks to the "classical" classification of the $S L(2, \mathbb{R})$-homogeneous spaces, we are going to recognize the lightlike ones.

Proposition 2.1 (Classification of $S L(2, \mathbb{R})$-homogeneous spaces).

(1) Any $S L(2, \mathbb{R})$-homogeneous space is isomorphic to one of the following:

(a) The circle $S^{1}=S L(2, \mathbb{R}) / A f f(\mathbb{R})$ endowed with its natural projective structure.

(b) The hyperbolic plane $=S L(2, \mathbb{R}) / K$ with its Riemannian metric of constant negative curvature.

(c) The affine punctured plane $\mathbb{R}^{2} \backslash\{0\}=S L(2, \mathbb{R}) / N$ equipped with an affine flat connection, together with a lightlike metric.

(d) A Hopf affine torus $\mathbb{R}^{2} \backslash\{0\} /\{x \sim a x\}=S L(2, \mathbb{R}) / \Gamma_{0} . N$ endowed with a flat projective structure.

(e) A space $S L(2, \mathbb{R}) / \Gamma$, where $\Gamma$ is a discrete subgroup of $S L(2, \mathbb{R})$. It is locally an anti-de Sitter space, i.e. a Lorentz manifold with negative constant curvature.

(2) Up to homothety, the unique lightlike $S L(2, \mathbb{R})$-homogeneous spaces having a noncompact isotropy are:

(a) The lightcone $C \mathrm{Co}^{1}$, i.e. the circle $S^{1}$ endowed with the null metric.

(b) The lightcone $C^{2}$, namely $\mathbb{R}^{2} \backslash\{0\}$ endowed with the lightlike metric $d \theta^{2}$, where $\mathbb{R}^{2} \backslash\{0\}$ is parametrized by the polar coordinates $(r, \theta)$. 
Proof. The proof of the first part is standard; we just give details in the lightlike case.

Let $\Sigma$ be an $S L(2, \mathbb{R})$-homogeneous space of dimension $\geq 2$, i.e. $\Sigma \cong S L(2, \mathbb{R}) / H$, where $H$ is the stabilizer of some $p \in \Sigma$ which is conjugate, as shown above, to one of the following subgroups: $K, N, \Gamma_{0} N$ and $\Gamma$. Let $\mathfrak{h}$ be the Lie algebra of $H$. Considering the isotropy representation

$$
\rho_{H}: H \longrightarrow G L\left(T_{p}(\Sigma)\right)=G L(\mathfrak{g} / \mathfrak{h}),
$$

one observes that when $H=K$ or $\Gamma_{0} N$ (with $\Gamma_{0} \neq 1$ ), $\rho_{H}(H)$ is not conjugate to a subgroup of $O(0,1)$. Now if $H=\Gamma$, then $\rho_{H}(\Gamma)$ is conjugate to a subgroup of $O(1,2)$. This is just because the Killing form on $\mathfrak{s l}(2, \mathbb{R})$ has Lorentz signature. If moreover $\rho_{H}(\Gamma)$ is conjugate to a subgroup of $O(0,2)$, then $\rho_{H}(\Gamma)$ has to be finite. Since the kernel of the adjoint representation of $S L(2, \mathbb{R})$ is finite, we get that $\Gamma$ is finite. Therefore the unique lightlike $S L(2, \mathbb{R})$-homogeneous space of dimension $\geq 2$ with noncompact isotropy is $\mathbb{R}^{2} \backslash\{0\}$.

In order to check that the lightlike metric has the form $\alpha d \theta^{2}$ for some $\alpha \in \mathbb{R}_{+}^{*}$, one argues as follows. We consider the basis $X, Y, Z$ of $S L(2, \mathbb{R})$ introduced in 2.2.1. By a slight abuse of language, they will also denote vector fields of $\mathbb{R}^{2} \backslash\{0\}$ induced by the $S L(2, \mathbb{R})$-action. At $p=(1,0)$, the vector $X$ is the unique nontrivial eigenspace of $\rho_{N}$, and thus the orbit of $p$ by the flow $\phi_{X}^{t}$ must coincide with the null leaf $\mathcal{N}_{(1,0)}$, which is therefore a radial half-line. The other null leaves are also radial, since they are images of $\mathcal{N}_{(1,0)}$ by the $S L(2, \mathbb{R})$-action. By homogeneity, the metric must have the form $\alpha d \theta^{2}$.

Remark 2.2. Proposition 2.1 is a special case of Theorem 1.3, where $G=O(1,2)$.

For later use, let us state the following fact which follows directly from the previous description of the lightlike surface $\mathbb{R}^{2} \backslash\{0\}$ (here, $X, Y, Z$ is the basis of $S L(2, \mathbb{R})$ introduced in 2.2 .17 .

Fact 2.3. If $Y$ is isotropic at some $p \in \mathbb{R}^{2} \backslash\{0\}$, then $Y$ vanishes at $p$ and $X$ is isotropic at $p$.

2.3. Generalities on semisimple groups; notation. [See for instance [14, chapters II and VI]. Let $G$ be a semisimple group acting isometrically on $(M, h)$. This means that we have a smooth homomorphism $\rho: G \rightarrow D i f f^{\infty}(M)$ such that for every $g \in G, \rho(g)$ preserves $h$, i.e. $\rho(g)^{*} h=h$. Let $\mathfrak{g}$ be the Lie algebra of $G$. For any $X$ in $\mathfrak{g}$, we will generally use the notation $\phi_{X}^{t}$ instead of $\rho(\exp (t X))$. By a slight abuse of language, we will also denote by $X$ the vector field of $M$ generated by the flow $\phi_{X}^{t}$.

We get for every $p \in M$ a homomorphism $\lambda_{p}: \mathfrak{g} \rightarrow T_{p} M$, defined by $\lambda_{p}(X)=X_{p}$. The flow $\phi_{X}^{t}$ stabilizes $p$ iff $X_{p}=0$, and we denote by $\mathfrak{g}_{p}$ the Lie algebra of the stabilizer of $p$.

We say that $X \in \mathfrak{g}$ is lightlike at $p \in M$ (or isotropic) (resp. spacelike) if $h_{p}\left(X_{p}, X_{p}\right)=0\left(\operatorname{resp} . h_{p}\left(X_{p}, X_{p}\right)>0\right)$.

We denote by $\mathfrak{s}_{p}$ the subspace of all vectors of $\mathfrak{g}$ which are isotropic at $p \in M$.

Let $O$ be a lightlike $G$-orbit of some $p \in M$, that is, $O \cong G / G_{p}$, where $G_{p}$ is the stabilizer of $p$. The tangent space $T_{p} O$ is identified by $\lambda_{p}$ to the quotient $\mathfrak{g} / \mathfrak{g}_{p}$. In fact the isotropy representation on $T_{p} O$ is equivalent to the adjoint representation $A d$ of $G_{p}$ on $\mathfrak{g} / \mathfrak{g}_{p}$. In particular $G_{p}$ is mapped, up to conjugacy, to a subgroup of $O(0, n)$. 
Similarly, the Euclidean space $T_{p} O / \mathcal{N}_{p}$ is identified to $\mathfrak{g} / \mathfrak{s}_{p}$, where $A d: G_{p} \longrightarrow$ $G L\left(\mathfrak{g} / \mathfrak{s}_{p}\right)$ preserves a positive definite inner product on $\mathfrak{g} / \mathfrak{s}_{p}$ so that $G_{p}$ acts on $\mathfrak{g} / \mathfrak{s}_{p}$ by orthogonal matrices. In particular, if we consider the tangent representation $a d: \mathfrak{g}_{p} \longrightarrow \operatorname{End}\left(\mathfrak{g} / \mathfrak{s}_{p}\right)$, the Lie subalgebra $\mathfrak{g}_{p}$ acts by skew symmetric matrices on $\mathfrak{g} / \mathfrak{s}_{p}$. We will use the same notation for the elements of the quotients $\mathfrak{g} / \mathfrak{g}_{p}$ and $\mathfrak{g} / \mathfrak{s}_{p}$ and their representatives in the Lie algebra $\mathfrak{g}$.

We fix once and for all a Cartan involution $\Theta$ on the Lie algebra $\mathfrak{g}$. This yields a Cartan decomposition $\mathfrak{g}=\mathfrak{k} \oplus \mathfrak{p}, \mathfrak{k}$ (resp. $\mathfrak{p}$ ) being the eigenspace of $\Theta$ associated with the eigenvalue +1 (resp. -1 ).

We choose $\mathfrak{a}$ as a maximal abelian subalgebra of $\mathfrak{p}$ and denote $\mathfrak{m}$ as the centralizer of $\mathfrak{a}$ in $\mathfrak{k}$. This choice yields a rootspace decomposition of $\mathfrak{g}$, namely there is a finite family $\Sigma^{+}=\left\{\alpha_{1}, \ldots, \alpha_{s}\right\}$ of nonzero elements of $\mathfrak{a}^{*}$ such that $\mathfrak{g}=\bigoplus_{\alpha \in \Sigma^{+}} \mathfrak{g}_{-\alpha} \oplus$ $\mathfrak{g}_{0} \oplus \bigoplus_{\alpha \in \Sigma^{+}} \mathfrak{g}_{\alpha}$. For every $X \in \mathfrak{a}, a d_{X}(Y)=\alpha(X) Y$ as soon as $Y \in \mathfrak{g}_{\alpha}$. The Lie subalgebra $\mathfrak{g}_{0}$ is in the kernel of $a d_{X}$ for every $X \in \mathfrak{a}$ and splits as a sum: $\mathfrak{g}_{0}=\mathfrak{a} \oplus \mathfrak{m}$.

The positive Weyl chamber $\mathfrak{a}^{+} \subset \mathfrak{a}$ contains those $X \in \mathfrak{a}$ such that $\alpha(X) \geq 0$ for all $\alpha \in \Sigma^{+}$. Its image by the exponential map is denoted by $A^{+}$. Let $\Sigma^{-}=$ $\left\{-\alpha_{1}, \ldots,-\alpha_{s}\right\}$.

The stable subalgebra (for $\mathfrak{a}$ ) $W^{s}=\bigoplus_{\alpha \in \Sigma^{-}} \mathfrak{g}_{\alpha}$ and the unstable one $W^{u}=$ $\bigoplus_{\alpha \in \Sigma^{+}} \mathfrak{g}_{\alpha}$ are both nilpotent subalgebras of $\mathfrak{g}$ mapped diffeomorphically by the exponential map of $\mathfrak{g}$ onto two subgroups $N^{+} \subset G$ and $N^{-} \subset G$.

Given $X \in \mathfrak{a}$, its stable algebra is $W_{X}^{s}=\bigoplus_{\alpha(X)<0} \mathfrak{g}_{\alpha}$ and its unstable algebra is $W_{X}^{u}=\bigoplus_{\alpha(X)>0} \mathfrak{g}_{\alpha}$.

Let us now prove a lemma which will be useful in the sequel:

Lemma 2.4. The subalgebra $W_{X}^{s}$ has the following properties:

(1) $\left[\mathfrak{g}, W_{X}^{s} \cap \mathfrak{g}_{p}\right] \subset \mathfrak{s}_{p}$.

(2) $\left[\mathfrak{s}_{p}, W_{X}^{s} \cap \mathfrak{g}_{p}\right] \subset \mathfrak{g}_{p}$.

Proof. Let $Y \in W_{X}^{s} \cap \mathfrak{g}_{p}$.

(1) Since $Y \in \mathfrak{g}_{p}, a d_{Y}$ acts on $\mathfrak{g} / \mathfrak{s}_{p}$ by a skew symmetric endomorphism, which is moreover nilpotent since $Y \in W_{X}^{s}$. Hence $a d_{Y}$ acts by the null endomorphism on $\mathfrak{g} / \mathfrak{s}_{p}$, which means that $a d_{Y}$ maps $\mathfrak{g}$ to $\mathfrak{s}_{p}$.

(2) $a d_{Y}$ acts as a nilpotent endomorphism of $\mathfrak{g} / \mathfrak{g}_{p}$ (identified with the tangent space) and has $\mathfrak{s}_{p} / \mathfrak{g}_{p}$ (identified to the isotropic direction) as a 1dimensional eigenspace. By nilpotency the action on it is trivial, i.e. $a d_{Y}$ maps $\mathfrak{s}_{p}$ into $\mathfrak{g}_{p}$.

Finally, recall that a semisimple Lie group of finite center admits a Cartan decomposition $G=K A K$, where $K$ is a maximal compact subgroup of $G$.

2.4. Nonproper actions. (See for instance 15] for a recent survey about these notions.)

Definition 2.5. Let $G$ act on $M$. A sequence $\left(p_{k}\right)$ is nonescaping if there is a sequence of transformations $g_{k} \in G$ such that both $\left(p_{k}\right)$ and $\left(q_{k}\right)=\left(g_{k}\left(p_{k}\right)\right)$ lie in a compact subset of $M$ but $\left(g_{k}\right)$ tends to $\infty$ in $G$, i.e. leaves any compact set of $G$.

- The sequence $\left(g_{k}\right)$ is called a return sequence for $\left(p_{k}\right)$.

- In the sequel, we will sometimes assume that $\left(p_{k}\right)$ and $\left(q_{k}\right)$ converge to $p$ and $q$ in $M$.

One says that the group $G$ acts nonproperly if it admits a nonescaping sequence. 
Here is a nice criterion for actions of semisimple Lie groups of finite center to be nonproper:

Lemma 2.6. Let $G$ be a noncompact semisimple group with finite center. Then $G$ acts nonproperly iff any Cartan subgroup $A$ acts nonproperly.

Proof. $G$ admits a Cartan decomposition $K A K$, where $K$ is compact. Let $\left(p_{k}\right)$ be a nonescaping sequence of the $G$-action and $\left(g_{k}\right)$ its return sequence. Write $g_{k}=l_{k} a_{k} r_{k} \in K A K$. Then, $p_{k}^{\prime}=r_{k}\left(p_{k}\right)$ is a nonescaping sequence for the $A$ action, with associated return sequence $\left(a_{k}\right)$. Obviously $\left(a_{k}\right)$ goes to infinity in $A$ since $\left(g_{k}\right)$ goes to infinity in $G$.

\section{A Key FACT ON THE STABLE SPACE}

Here we state a crucial ingredient for the proofs of all our theorems. In all that follows, $G$ is a noncompact semisimple Lie group with finite center acting locally faithfully, nonproperly and isometrically on a lightlike manifold $(M, h)$. The main result of this section is:

Proposition 3.1. If no factor of $G$ is locally isomorphic to $S L(2, \mathbb{R})$, there exists a Cartan subalgebra $\mathfrak{a}_{0}$ such that for some $X_{0} \in \mathfrak{a}_{0}$ and $p_{0} \in M$, both $X_{0}$ and its stable algebra $W_{X_{0}}^{s}$ are isotropic at $p_{0}$.

3.1. Starting fact. The nonproperness of the action of $G$ leads to a fundamental fact, already observed in [16] for Lorentzian metrics, which is the existence of $p \in M$ and $X \in \mathfrak{a}$ such that $W_{X}^{s}$ is isotropic at $p$. Let us recall its proof.

Proposition 3.2 ([16]). Let $\mathfrak{a}$ be a Cartan subalgebra of $\mathfrak{g}$.

(1) If the flow of $X \in \mathfrak{a}$ acts nonproperly, then for any sequence $p_{k} \rightarrow p$ which is nonescaping for the action of $\phi_{X}^{t}$, the stable space $W_{X}^{s}$ is isotropic at $p$.

(2) More generally, if $p_{k} \rightarrow p$ is a nonescaping sequence for the A-action, then there exists $X \in \mathfrak{a}$ such that $W_{X}^{s}$ is isotropic at $p \in M$.

Proof. (1) Denote $\phi_{X}^{t}=\exp (t X)$ as the flow of $X$, and let $\left(t_{k}\right)$ be a return time sequence for $\left(p_{k}\right)$, i.e. $\phi_{X}^{t_{k}}$ is a return sequence of $p_{k}$, which means that $q_{k}=\phi_{X}^{t_{k}}\left(p_{k}\right)$ stay in a compact subset of $M$.

Let $Y \in \mathfrak{g}_{\alpha}$; then $[X, Y]=\alpha(X) Y$. Hence for any $x \in M, D_{x} \phi_{X}^{t} Y_{x}=$ $e^{t \alpha(X)} Y_{\phi_{X}^{t}(x)}$. Assume that $\alpha(X)<0$. Then

$$
h_{p_{k}}\left(Y_{p_{k}}, Y_{p_{k}}\right)=h_{q_{k}}\left(D_{p_{k}} \phi_{X}^{t_{k}}\left(Y_{p_{k}}\right), D_{p_{k}} \phi_{X}^{t_{k}}\left(Y_{p_{k}}\right)\right)=e^{2 t_{k} \alpha(X)} h_{q_{k}}\left(Y_{q_{k}}, Y_{q_{k}}\right) .
$$

On the left hand side, passing to the limit yields $h_{p}\left(Y_{p}, Y_{p}\right)$.

On the right hand side, since $\left(q_{k}\right)$ lie in a compact set, $h_{q_{k}}\left(Y_{q_{k}}, Y_{q_{k}}\right)$ is bounded. Therefore, since $\alpha(X)<0$, this right hand term tends to 0 , yielding $h_{p}\left(Y_{p}, Y_{p}\right)=0$. This proves that $W_{X}^{s}$ is isotropic at $p$.

(2) Let $\left(X_{k}\right)$ be a sequence in $\mathfrak{a}$ such that $\exp \left(X_{k}\right)$ is a return sequence for $\left(p_{k}\right)$. Let $\|$.$\| be a Euclidean norm on \mathfrak{a}$ and, considering if necessary a subsequence, assume $\left(\frac{X_{k}}{\left\|X_{k}\right\|}\right)$ converges to some $X \in \mathfrak{a}$. As above, one proves that $W_{X}^{s}$ is isotropic at $p$.

Remark 3.3. This result is nothing but a generalization of the linear (pointwise) easy fact: if a matrix $A$ preserves a lightlike scalar product, then its corresponding stable and unstable spaces are isotropic. In our particular case, if $X \in \mathfrak{a} \cap \mathfrak{g}_{p}$, i.e. $X$ stabilizes $p$, then both $W_{X}^{s}$ and $W_{X}^{u}$ are isotropic at $p$. 
3.2. Proof of Proposition 3.1, The proof follows from several observations. The simplest one is that for lightlike metrics (in contrast with the Lorentz case), the isotropic direction is unique on each tangent space $T_{p} M$. Furthermore, it coincides with the nontrivial eigenspace (if any) of any infinitesimal isometry fixing $p$. The hypothesis made in Proposition 3.1 that $G$ has no factor locally isomorphic to $S L(2, \mathbb{R})$ will only be used in Lemma 3.7 .

Lemma 3.4. For any $p \in M$, the subspace of isotropic vectors $\mathfrak{s}_{p}$ is a Lie subalgebra of $\mathfrak{g}$.

Proof. Let $X, Y \in \mathfrak{s}_{p}$, and let $\phi_{X}^{t}$ be the isometric flow generated by $X$ on $M$. Then

$$
[X, Y]_{p}=\lim _{t \rightarrow 0} \frac{1}{t}\left[d \phi_{X}^{-t}\left(Y_{\phi_{X}^{t}(p)}\right)-Y_{p}\right]
$$

Since $X, Y$ are isotropic at $p$, their integral curves at $p$ are supported by the null leaf $\mathcal{N}_{p}$ and thus $Y_{\phi_{X}^{t}(p)}$ is isotropic. Because $\phi_{X}^{-t}$ is an isometry, $d \phi_{X}^{-t}\left(Y_{\phi_{X}^{t}(p)}\right)$ is also isotropic. Together with the observation made above, this yields the lemma.

Lemma 3.5. $G$ stabilizes no $p \in M$.

Proof. Suppose by contradiction that $G$ stabilizes $p \in M$. Then $G$ acts on $T_{p} M$ by $\rho: g \mapsto d_{p} g \in G L\left(T_{p} M\right)$. Since $G$ preserves the lightlike scalar product $h_{p}$, it is mapped by $\rho$ into a subgroup of $O(0, n)$. Thus, at the level of Lie algebras, we get a homomorphism $d \rho: \mathfrak{g} \rightarrow \mathfrak{o}(0, n)$. Now, we prove:

Sublemma 3.6. Any homomorphism from $\mathfrak{g}$ to $\mathfrak{o}(0, n)$ is trivial.

Proof. Without loss of generality, we can assume that $\mathfrak{g}$ is simple. Let $\lambda$ be a homomorphism from $\mathfrak{g}$ to $\mathfrak{o}(0, n)$, and let $\pi$ be the projection from $\mathfrak{o}(0, n)$ to $\mathfrak{o}(n)$. Consider the homomorphism $\lambda \circ \pi: \mathfrak{g} \longrightarrow \mathfrak{o}(n)$. Since $\mathfrak{g}$ is simple and noncompact, it has no nontrivial homomorphism into the Lie algebra of a compact group; this implies that $\lambda \circ \pi$ is trivial. So, $\mathfrak{g}$ is mapped by $\lambda$ into the kernel $\mathfrak{g}_{0}$ of $\pi$, that is, the algebra of matrices of the form $\left(\begin{array}{cccc}\mu & x_{1} & \ldots & x_{n} \\ 0 & & & \\ \cdot & & 0 & \\ \cdot & & & \end{array}\right)$. Since $\mathfrak{g}_{0}$ is solvable and $\mathfrak{g}$ is simple, we conclude that $\lambda$ is trivial.

As a corollary, the $\rho$-image of any connected compact subgroup $K \subset G$ is trivial. However such $K$ preserves a Riemannian metric. But on a connected manifold $M$, a Riemannian isometry which fixes a point and has a trivial derivative at this point must be the identity on $M$. This is easily seen since in the neighborhood of any fixed point a Riemannian isometry is linearized by the exponential map. Hence $K$ acts trivially on $M$, and therefore $G$ does not act faithfully, which contradicts our hypothesis and completes the proof of our lemma.

Lemma 3.7. If $G$ has no factor locally isomorphic to $S L(2, \mathbb{R})$, then no Cartan subalgebra $\mathfrak{a}$ meets the stabilizer subalgebra: $\mathfrak{a} \cap \mathfrak{g}_{p}=\{0\}$ for any $p \in M$.

Proof. Assume by contradiction that $\mathfrak{a} \cap \mathfrak{g}_{p} \neq\{0\}$, and let us take $X \neq 0$ in this intersection. Apply Remark 3.3 to $X$ to get that the subspaces $W_{X}^{s}$ and $W_{X}^{u}$ are both isotropic at $p$. It is a general fact that the Lie subalgebra $\mathfrak{n}$ generated by $W_{X}^{s}$ and $W_{X}^{u}$ is an ideal of $\mathfrak{g}$ (see for instance [16]), hence is a factor of $\mathfrak{g}$. It acts on the 
1-dimensional manifold $\mathcal{N}_{p}$. This action is faithful. Otherwise its kernel $\mathfrak{s}$ would be the Lie algebra of a semisimple group $S \subset G$ which would have fixed points on $M$, in contradiction with Lemma 3.5. Now, the only semisimple algebra acting faithfully on a 1 -dimensional manifold is $\mathfrak{s l}(2, \mathbb{R})$. This contradicts our hypothesis that $\mathfrak{g}$ has no such factor.

Lemma 3.8. Let $H$ be a Lie group with Lie algebra $\mathfrak{s l}(2, \mathbb{R})$.

(1) If $H$ is linear, then it is isomorphic to either $S L(2, \mathbb{R})$ or $P S L(2, \mathbb{R})$.

(2) If $H$ is a subgroup of a Lie group $G$ with finite center, then it is a finite covering of $P S L(2, \mathbb{R})$.

Proof. The point is that all the representations of the Lie algebra $\mathfrak{s l}(2, \mathbb{R})$ integrate to actions of the group $S L(2, \mathbb{R})$ itself, and not merely its universal cover. Indeed all the irreducible representations are isomorphic to symmetric powers of the standard representation, or equivalently to representations on spaces of homogeneous polynomials of a given degree, in two variables $x$ and $y$ (see for instance 14, section I.9). Clearly, $S L(2, \mathbb{R})$ acts on these polynomials, and $P S L(2, \mathbb{R})$ acts iff the degree is even. For the last point, observe that the adjoint representation of $G$ has finite kernel.

End of the proof of Proposition 3.1, From Proposition 3.2, there exist $X \in \mathfrak{a}$ and $p \in M$ such that $W_{X}^{s}$ is isotropic at $p$. Since $\mathfrak{g}$ has no local factor isomorphic to $\mathfrak{s l}(2, \mathbb{R})$, we have $\operatorname{dim} W_{X}^{s}>1$ (otherwise the subalgebra $\mathfrak{a} \oplus \Sigma_{\alpha(X) \geq 0} \mathfrak{g}_{\alpha}$ is supplementary to $W_{X}^{s}$ and would have codimension 1, yielding an action of $\mathfrak{g}$ on a 1-dimensional manifold). For a lightlike metric, an isotropic space has dimension at most 1 , so that the evaluation of $W_{X}^{s}$ at $p$ has at most dimension 1 and thus $W_{X}^{s}$ contains at least a nonzero vector $Y_{0}$ vanishing at $p$.

By the Jacobson-Morozov Theorem (see [14, Theorem 10.3), the nilpotent element $Y_{0}$ belongs to some subalgebra $\mathfrak{h}$ isomorphic to $\mathfrak{s l}(2, \mathbb{R})$, i.e. generated by an $\mathfrak{s l}_{2}$-triple $\left\{X_{0}, Y_{0}, Z_{0}\right\}$, such that $\left[X_{0}, Y_{0}\right]=2 Y_{0},\left[X_{0}, Z_{0}\right]=-2 Z_{0}$, and $\left[Y_{0}, Z_{0}\right]=X_{0}$.

Let $H \subset G$ be the group associated to $\mathfrak{h}$. From the lemma above and the fact that $G$ has finite center, $H$ is a finite covering of $P S L(2, \mathbb{R})$. Let us call $\Sigma$ the $H$-orbit of $p$. Because in any finite-dimensional representation of $\mathfrak{s l}(2, \mathbb{R})$ any $\mathbb{R}$ split element is mapped on some $\mathbb{R}$-split element, the Cartan subalgebra $\mathbb{R} X_{0}$ is contained in a Cartan subalgebra $\mathfrak{a}_{0}$ of the ambient algebra $\mathfrak{g}$. By Lemma 3.7, we know that $X_{0}$ does not vanish at $p$. On the other hand $Y_{0}$ vanishes at $p$, which implies $\mathbb{R} Y_{0}=\mathfrak{g}_{p}$. In particular, $\Sigma$ is 2-dimensional and cannot be Riemannian because $Y_{0} \in \mathfrak{g}_{p}$ shows that the action of $H$ is nonproper on $\Sigma$. We obtain the fact that $\Sigma$ is a lightlike surface homothetic, up to finite cover, to $\left(\mathbb{R}^{2} \backslash\{0\}, d \theta^{2}\right)$. We already mentioned that $H$ acts nonproperly on $\Sigma$. The group $\exp \left(\mathbb{R} X_{0}\right)$ is a Cartan subgroup of $H$, and by Lemma 2.6] $\exp \left(\mathbb{R} X_{0}\right)$ also acts nonproperly on $\Sigma$. Thus we can find $\left(q_{k}\right)$ as both a sequence of $\Sigma$ converging to $p_{0} \in \Sigma$ and a sequence of return times $\left(t_{k}\right)$, such that $h_{k} \cdot q_{k}$ converges in $\Sigma$, where $h_{k}=\exp \left(t_{k} X_{0}\right)$. Now we apply the first part of Proposition 3.2 to $X_{0}$ and $\mathfrak{a}_{0}$, and deduce that $W_{X_{0}}^{s}$ is isotropic at $p_{0}$ (where $W_{X_{0}}^{s}$ is defined relatively to $\mathfrak{a}_{0}$ ). In particular $Y_{0}$ is isotropic at $p_{0}$, and Fact 2.3 then ensures that $X_{0}$ is also isotropic at $p_{0}$. 


\section{Proof of Theorem 1.3}

4.1. Reduction lemma. The following fact together with Proposition 3.1 reduces the proof of Theorem 1.3 to the case of nonproper transitive actions of semisimple groups.

Lemma 4.1 (Reduction to the transitive case). Let $G$ be a semisimple Lie group with finite center and no factor locally isomorphic to $S L(2, \mathbb{R})$, acting faithfully nonproperly isometrically on a lightlike manifold. At any $p \in M$ for which there exists $X$ such that $W_{X}^{s}$ is isotropic at $p$, the orbit $G . p$ is lightlike and $G$ acts nonproperly on it. In fact, the stabilizer subalgebra $\mathfrak{g}_{p}$ contains nilpotent elements.

Proof. Let $p \in M$ and let $X$ be such that $W_{X}^{s}$ is isotropic at $p$. We already saw at the end of the proof of Proposition 3.1 that $W_{X}^{s}$ has dimension $>1$. If it is isotropic at $p$, then it contains a nonzero element $Y_{0} \in W_{X}^{s} \cap \mathfrak{g}_{p}$ vanishing at $p$. But $Y_{0}$ is a nonzero nilpotent element in $\mathfrak{g}$, and in particular $\operatorname{Ad}\left(\exp \left(t Y_{0}\right)\right)$ is noncompact, proving that the stabilizer of $p$ is noncompact. Therefore the action of $G$ on the $G$-orbit $G . p$ is nonproper, and $\mathfrak{g}_{p}$ contains nilpotent elements.

Let us show that G.p is lightlike. Lemma 3.5 shows that G.p cannot be reduced to $p$. Suppose now by contradiction that G.p is Riemannian. Then any vector which is isotropic at $p$ must vanish there, in particular $W_{X}^{s} \subset \mathfrak{g}_{p}$.

Consider the infinitesimal action of $Y_{0}$ on the tangent space of the orbit at $p$. This action is just $a d_{Y_{0}}: \mathfrak{g} / \mathfrak{g}_{p} \longrightarrow \mathfrak{g} / \mathfrak{g}_{p}$. If $G$.p is supposed to be Riemannian, it is at the same time skew symmetric and nilpotent, hence trivial on $\mathfrak{g} / \mathfrak{g}_{p}$, which means that $a d_{Y_{0}}(\mathfrak{g}) \subset \mathfrak{g}_{p}$. Now, use the Jacobson-Morozov theorem to get an $\mathfrak{s l}(2, \mathbb{R})$-triple $\left\{Z_{0}, X_{0}, Y_{0}\right\}$. Then $a d_{Y_{0}}\left(Z_{0}\right)=X_{0}$, so that $X_{0} \in \mathfrak{g}_{p}$. Since we already saw that $X_{0}$ is in a Cartan subalgebra of $\mathfrak{g}$, this yields a contradiction with Lemma 3.7.

4.2. Proof in the simple case. We now give the proof of Theorem 1.3 assuming that the group $G$ is simple with finite center, not locally isomorphic to $S L(2, \mathbb{R})$, and the action is transitive and nonproper. The general case of semisimple groups will be handled in the next section. The proof will be achieved in several steps (let us mention that some of the arguments below are similar to those in [9]).

Step 1. There exist $p \in M$ and $X$ in some Cartan subalgebra a such that $W_{X}^{s} \subset \mathfrak{g}_{p}$.

Proof. Proposition 3.1 says that for some $p \in M$, there exists $X$ in a Cartan subalgebra of $\mathfrak{g}$ such that both $X$ and $W_{X}^{s}$ are isotropic at $p$. For any $Y$ in $W_{X}^{s}$, the Lie algebra generated by $X$ and $Y$ is isomorphic to the Lie algebra $\mathfrak{a f f}(\mathbb{R})$ and acts on the null leaf $\mathcal{N}_{p}$. Up to isomorphism, there are exactly two actions of $\mathfrak{a f f}(\mathbb{R})$ on a connected 1-dimensional manifold:

(1) The usual affine action of $\mathfrak{a f f}(\mathbb{R})$ on the line. For this action, a conjugate of $X$ vanishes somewhere.

(2) The nonfaithful action, for which $Y$ acts trivially.

The first case cannot occur without contradicting Lemma 3.7, so that only possibility (2) occurs and thus $W_{X}^{s} \subset \mathfrak{g}_{p}$.

Step 2. The $\mathbb{R}$-rank of $\mathfrak{g}$ equals 1 .

Proof. Suppose the $\mathbb{R}$-rank of $\mathfrak{g}$ is greater than 1 . Let $\alpha$ be a root such that $\alpha(X)>0$ and $\beta$ be an adjacent root in the Dynkin diagram, according to the choice of a basis $\Phi$ of positive simple roots for which $\gamma \in \Phi \Longrightarrow \gamma(X) \geq 0$. (See 
[14, $\mathrm{p}$ 160.) By definition, $\alpha+\beta$ is also a root and $(\alpha+\beta)(X)>0$, which means that $\mathfrak{g}_{-\alpha}$ and $\mathfrak{g}_{-(\alpha+\beta)}$ are different and contained in $W_{X}^{s}$.

Let $T_{\alpha}$ and $T_{\alpha+\beta}$ be the vectors of $\mathfrak{a}$ dual to $\alpha$ and $\alpha+\beta$, respectively. They are linearly independent. Moreover, $T_{\alpha} \in\left[\mathfrak{g}_{\alpha}, \mathfrak{g}_{-\alpha}\right] \subset a d_{\mathfrak{g}}\left(W_{X}^{s}\right)(\mathfrak{g})$, and the same is true for $T_{\alpha+\beta}$,

By the first step and Lemma 2.4, $T_{\alpha}$ and $T_{\alpha+\beta}$ are isotropic at $p$. Hence, there is a nontrivial linear combination of them which vanishes at $p$. This contradicts Lemma 3.7 claiming that $\mathfrak{a} \cap \mathfrak{g}_{p}=\{0\}$. Therefore, $\mathfrak{g}$ has rank 1 .

Remark 4.2. It is exactly here that we need $G$ to be simple!

Step 3. The Lie algebra $\mathfrak{g}$ is isomorphic to $\mathfrak{o}(1, n)$.

Proof. Suppose that $\mathfrak{g}$ is not isomorphic to $\mathfrak{o}(1, n)$; then we have two roots $\alpha$ and $2 \alpha$ such that $\alpha(X)>0$.

Claim 4.3. The bracket $\left[\mathfrak{g}_{2 \alpha}, \mathfrak{g}_{-\alpha}\right] \neq 0$.

Let us continue the proof assuming the claim. Consider a nonzero $Y \in\left[\mathfrak{g}_{2 \alpha}, \mathfrak{g}_{-\alpha}\right]$ $\subset \mathfrak{g}_{\alpha}$. By Lemma 2.4 $Y$ is isotropic at $p$. Let $\Theta$ be the Cartan involution (see 14 p. 355); then $\Theta Y \in W_{X}^{s}$, and hence belongs to $\mathfrak{g}_{p}$, by Step 1. Lemma 2.4 then implies that $[Y, \Theta Y] \in \mathfrak{g}_{p}$, in particular $\mathfrak{a} \cap \mathfrak{g}_{p} \neq 0$, which contradicts Lemma 3.7.

Proof of the claim. The rank 1 simple Lie groups of noncompact type are known to be the isometry groups of symmetric spaces of negative curvature, namely the real, complex and quaternionic hyperbolic spaces, together with the hyperbolic Cayley plane. A direct computation can be performed to prove the claim. Let us give another synthetic proof. By contradiction, if $\left[\mathfrak{g}_{2 \alpha}, \mathfrak{g}_{-\alpha}\right]=0$, the sum $\mathfrak{l}=$ $\mathfrak{g}_{0}+\mathfrak{g}_{-\alpha}+\mathfrak{g}_{-2 \alpha}+\mathfrak{g}_{2 \alpha}$ would be a subalgebra of $\mathfrak{g}$. For the sake of simplicity, let us work with groups instead of algebras. Let $L$ be the group associated to our last subalgebra $\mathfrak{l}$. Clearly, $L$ is noncompact. The point is that there is a dichotomy for noncompact connected isometry subgroups of negatively curved symmetric spaces. If they have a nontrivial solvable radical, then they fix a point at infinity and thus are contained in a parabolic group. In particular, they have a compact simple Levi part (see [11). If not, the group is semisimple. It is clear that our $L$ contains a noncompact semisimple group, and therefore by the dichotomy it is semisimple. But in this case, $L$ will have a "symmetric" root decomposition, i.e. the negative of a root is a root, too. Thus, there must exist a nontrivial root space corresponding to $\alpha$, which contradicts the definition of $\mathfrak{l}$.

Step 4. The full isotropic subalgebra is $\mathfrak{s}_{p}=\mathfrak{a} \oplus \mathfrak{m} \oplus \mathfrak{g}_{-\alpha}$.

Proof. Recall that $\mathfrak{m}$ is the Lie algebra of the centralizer of $\mathfrak{a}$ in the maximal compact $K$. Since $\mathfrak{m} \subset\left[\mathfrak{g}_{\alpha}, \mathfrak{g}_{-\alpha}\right]$, Lemma 2.4 implies that it is isotropic at $p$.

On the other hand, if $Y \in \mathfrak{g}_{\alpha}$ is isotropic at $p$, Lemma 2.4 implies that the semisimple element $[Y, \Theta Y] \in\left[\mathfrak{g}_{-\alpha}, \mathfrak{g}_{\alpha}\right] \subset\left[\mathfrak{s}_{p}, W_{p}^{s} \cap \mathfrak{g}_{p}\right]$ is in the stabilizer subalgebra of $p$, which contradicts Lemma 3.7. Therefore, the isotropic subalgebra is exactly $\mathfrak{s}_{p}=\mathfrak{a} \oplus \mathfrak{m} \oplus \mathfrak{g}_{-\alpha}$.

Step 5. The full stabilizer subalgebra is $\mathfrak{g}_{p}=\mathfrak{m} \oplus \mathfrak{g}_{-\alpha}$. 
Proof. Every $Z \in \mathfrak{m}$ is isotropic at $p$. Suppose by contradiction that $Z \notin \mathfrak{g}_{p}$. Then there exists an element $Z+\lambda X \in \mathfrak{g}_{p}, \lambda \in \mathbb{R}^{*}$. We let it act on the normal space of the null leaf.

The action of $X$ on $\mathfrak{g} / \mathfrak{s}_{p}$ is identified to its action on $\mathfrak{g}_{\alpha}$ by the previous step. In particular the $X$-action has nonzero real eigenvalues.

The action of $\mathfrak{m}$ on $\mathfrak{g} / \mathfrak{s}_{p}$ has purely imaginary eigenvalues, since $\mathfrak{m}$ is contained in the Lie algebra of a maximal compact group. This is particularly true for the adjoint action of $Z$.

On the one hand, since $X$ and $Z$ commute by definition of $\mathfrak{m}$, the action of $Z+\lambda X$ on $\mathfrak{g} / \mathfrak{s}_{p}$ must have eigenvalues with nontrivial real part.

On the other hand, $Z+\lambda X \in \mathfrak{g}_{p}$ acts as a skew symmetric endomorphism on $\mathfrak{g} / \mathfrak{s}_{p}$, and thus has only purely imaginary eigenvalues: a contradiction. This shows that $\mathfrak{m} \subset \mathfrak{g}_{p}$, but since $\mathfrak{a} \cap \mathfrak{g}_{p}=0$ and $\mathfrak{g}_{p} \subset \mathfrak{s}_{p}$, we infer from the previous step the equality $\mathfrak{g}_{p}=\mathfrak{m} \oplus \mathfrak{g}_{-\alpha}$.

End. Since $\mathfrak{g}$ is isomorphic to $\mathfrak{o}(1, n)$ and the Lie algebra of the stabilizer $\mathfrak{g}_{p}$ is isomorphic to the Lie algebra of the group of Euclidean motions $E u c_{n}$, we conclude that $M$ is covered by $O(1, n) / E u c_{n}$ endowed with a left-invariant lightlike metric. All those metrics are homothetic to that of the lightcone in Minkowski space. Finally, because $n \geq 3$, the covering $O(1, n) / E u c_{n} \rightarrow M$ has to be finite, which completes the proof of Theorem 1.3 when $G$ is simple.

4.3. End of the proof. Thanks to Lemma 4.1 and Proposition 3.1, the complete proof of Theorem 1.3 reduces to the study of nonproper transitive actions of semisimple groups with no factor locally isomorphic to $S L(2, \mathbb{R})$. The work shown above will be useful thanks to the following reduction lemma:

Lemma 4.4 (Reduction to the simple case). Let $X$ be in a Cartan subalgebra of $\mathfrak{g}$, such that $W_{X}^{s}$ is isotropic at $p$. Consider the decomposition of $\mathfrak{g}$ in simple factors. Let $\mathfrak{h}$ be such a simple factor and let $H \subset G$ be the corresponding group. Suppose $X$ has a nontrivial projection on $\mathfrak{h}$. Then the $H$-orbit is nonproper and lightlike.

Proof. Write $\mathfrak{g}=\mathfrak{h}_{1} \oplus \ldots \oplus \mathfrak{h}_{s}$, where the $\mathfrak{h}_{i}$ 's are the simple factors of $\mathfrak{g}$, and call $X_{i}$ the projection of $X$ on $\mathfrak{h}_{i}$. If $W_{X_{i}, \mathfrak{h}_{i}}^{s}$ denotes the stable space of $X_{i}$ relative to $\mathfrak{h}_{i}$, it is straightforward to check that $W_{X}^{s}=W_{X_{1}, \mathfrak{h}_{1}}^{s} \oplus \ldots \oplus W_{X_{s}, \mathfrak{h}_{s}}^{s}$. In particular, if $W_{X}^{s}$ is isotropic at $p$ and $\mathfrak{h}$ is a simple factor on which $X$ has a nontrivial projection $X^{\prime}$, then $W_{X^{\prime}, \mathfrak{h}}^{s}$ is nontrivial and isotropic at $p$. We infer from Lemma 4.1 that the $H$-orbit of $p$ is lightlike and the action of $H$ on it is nonproper.

By this lemma, there is a simple factor $H$ of $G$ having a lightlike nonproper orbit $H$.p. It follows from the previous section that $H$ is locally isomorphic to $O(1, n)$, $n \geq 3$, and H.p is homothetic to $C o^{n}$ up to a finite cover. There is a semisimple group $H^{\prime}$ such that $G$ is a finite quotient of $H \times H^{\prime}$. This product still acts locally faithfully on $M$, so that we will assume $G=H \times H^{\prime}$ in the following. Consider $O=G$.p, the $G$-orbit containing H.p. The remaining part of Theorem 1.3 will follow from the geometric description of $O$ : up to a finite cover, it is a direct metric product $H . p \times H^{\prime} . p$. This is the content of the following proposition, which will also be useful when dealing with groups having factors locally isomorphic to $S L(2, \mathbb{R})$.

Proposition 4.5. Let $G$ be a semisimple Lie group acting locally faithfully transitively and nonproperly on a lightlike manifold $(M, h)$. We assume that $G=H \times H^{\prime}$, where $H$ is isomorphic to $O(1, n), n \geq 2$, and $H^{\prime}$ is semisimple. 
- We assume that $n \geq 3$. If for some $p \in M$ the orbit H.p is homothetic to $C o^{n}$, then $M$ is homothetic to a metric product $M=C o^{n} \times N$, where $N$ is an $H^{\prime}$-homogeneous Riemannian manifold.

- We assume that $n=2$. If for some $p \in M$ the orbit H.p is homothetic to $C o^{1}$, then $M$ is homothetic to a metric product $C o^{1} \times N$ as above. If H.p is homothetic to $C o^{2}$, then $M$ is a topological product $C o^{2} \times N$, and there is a finite-dimensional family of $G$-homogeneous lightlike metrics, which is, up to homothety, in natural one-to-one correspondence with the linear forms on $\mathbb{R}^{\operatorname{dim} N}$. Up to homothety, any such $h$ induces the standard lightlike metric on $\mathrm{Co}^{2}$ and an $\mathrm{H}^{\prime}$-homogeneous Riemannian metric on $N$.

In any case, the $G$ action is the product one.

Proof. $M$ is naturally foliated by lightcones $\mathcal{H}_{x}=H . x$. This foliation is $G$ invariant: if $g \in G$, then, $g \mathcal{H}_{x}=g H . x=H g . x=\mathcal{H}_{g . x}$, since $H$ is normal in $G$.

1) We first prove that $M$ is a topological product. By homogeneity it is sufficient to show that for $p \in M$, the intersection $H . p \cap H^{\prime} . p$ is reduced to $\{p\}$. If it were not the case, there would be $h^{\prime} \in H^{\prime}$ such that $h^{\prime} . p \in H . p$ and $h^{\prime} . p \neq p$. This would imply $h^{\prime} . H . p=H \cdot h^{\prime} \cdot p=H . p$. Hence $h^{\prime}$ would preserve the orbit H.p and act on it by commuting with $H$. We conclude thanks to the next lemma, which says that $h^{\prime}$ should act trivially on H.p, contradicting $h^{\prime} \cdot p \neq p$.

Lemma 4.6. Let $h^{\prime}$ be an isometry of the cone $C o^{n}, n \geq 2$ (resp. of $\left.C o^{1}\right)$, commuting with the action of $O(1, n)$ (resp. $O(1,2))$. Then $h^{\prime}$ is the identity map of $C o^{n}\left(\right.$ resp. Co $\left.{ }^{1}\right)$.

Proof. We begin with the case of $C o^{1}$. An isometry of $C o^{1}$ is just a diffeomorphism of $\mathbf{S}^{1}$. If such a diffeomorphism commutes with the projective action of $O(1,2)$ on $\mathbf{S}^{1}$, it must fix all the fixed points of parabolic elements in $O(1,2)$. But the set of these fixed points is precisely $\mathbf{S}^{1}$, so we are done.

In a higher dimension, we saw that writing $C o^{n}$ as $\mathbb{R} \times \mathbf{S}^{n-1}$ with the metric $0 \oplus g_{\mathbf{S}^{n-1}}$, the isometry $h^{\prime}$ is of the form $(t, x) \mapsto(t-\mu(x), \phi(x))$. Here $\phi$ is a conformal transformation of $\mathbf{S}^{n-1}$ satisfying $\phi^{*} g_{\mathbf{S}^{n-1}}=e^{2 \mu} g_{\mathbf{S}^{n-1}}$. If $h^{\prime}$ commutes with the action of $O(1, n)$, it must leave invariant any line of fixed points of parabolic elements in $O(1, n)$. This implies $\phi(x)=x$ for all $x$, and finally $\mu(x)=0$.

2) We now observe that $H^{\prime} \cdot p$ is Riemannian, because if it is not the case, it contains the null leaf $\mathcal{N}_{p}$ and we get $\mathcal{N}_{p} \subset H . p \cap H^{\prime} . p$, contradicting $H . p \cap H^{\prime} . p=$ $\{p\}$.

We first deal with the case $H=O(1, n), n \geq 3$. Let us suppose that H.p is homothetic to $C o^{n}$, and consider $S$ as a maximal compact subgroup in the isotropy group of $p$ in $H$. Since $H$ and $H^{\prime}$ commute, $S$ acts trivially on $H^{\prime} . p$ : $s h^{\prime} \cdot p=$ $h^{\prime} s . p=h^{\prime} . p$. By the first point of the proof, we know that $T_{p}(H . p)$ is transverse to $T_{p}\left(H^{\prime} . p\right)$. Moreover, there is an $S$-invariant splitting $T_{p} M=T_{p}\left(H^{\prime} . p\right) \oplus T_{p}\left(\mathcal{N}_{p}\right) \oplus E$, where $E$ is a Riemannian subspace of $T_{p}(H . p)$, on which $S$ acts irreducibly by the standard action of $O(n-1)$ on $\mathbb{R}^{n-1}$. Let us call $F$ the orthogonal of $T_{p}(H . p)$ in $T_{p} M$. This space is transverse to $E$ so that $F$ is the graph of a linear map $A: T_{p}\left(H^{\prime} . p\right) \oplus T_{p}\left(\mathcal{N}_{p}\right) \rightarrow E$. This map $A$ intertwines the trivial action of $S$ on $T_{p}\left(H^{\prime} . p\right) \oplus T_{p}\left(\mathcal{N}_{p}\right)$ with the irreducible one on $E$, so that $A=0$, and $F=$ $T_{p}\left(H^{\prime} . p\right) \oplus T_{p}\left(\mathcal{N}_{p}\right)$. As a consequence, the sum $T_{p}\left(H^{\prime} . p\right) \oplus T_{p}(H . p)$ is orthogonal 
for the metric $h_{p}$, and by homogeneity of $M$ this remains true at every point of $M$. The manifold $M$ is a metric product, $C o^{n} \times N$, where $N$ is Riemannian and $H^{\prime}$-homogeneous, as desired.

It remains to handle the case $H=O(1,2)$. If for some $p \in M, H$.p is homothetic to $C o^{1}$, i.e. it is 1-dimensional and lightlike, then clearly, $M$ is a metric product $C o^{1} \times N$, with $N$ Riemannian and $H^{\prime}$-homogeneous. Now, assume $H . p$ is homothetic to $C o^{2}$. Let us fix $\left(e_{1}, e_{2}\right)$ as a basis of $T_{p}(H . p)$, with $e_{1}$ lightlike, and $\left(e_{3}, \ldots, e_{s}\right)$ as a basis of $T_{p}\left(H^{\prime} . p\right)$. Let $H_{p}$ (resp. $H_{p}^{\prime}$ ) denote the stabilizer of $p$ in $H$ (resp. in $H^{\prime}$ ). Elements of $H_{p}$ induce transformations of $T_{p}(H . p)$ with matrices of the form $\left(\begin{array}{ll}1 & u \\ 0 & 1\end{array}\right), u \in \mathbb{R}$, in the basis $\left(e_{1}, e_{2}\right)$. In particular, if \langle\rangle is a Riemannian $H_{p}^{\prime}$-invariant scalar product on $T_{p}\left(H^{\prime} . p\right)$ and $l$ is any linear form on $T_{p}\left(H^{\prime} . p\right)$, then $h_{p}^{\prime}$, defined by $h_{p}^{\prime}\left(e_{1}, e_{i}\right)=0$ for all $i=\{1, \ldots, s\}, h_{p}^{\prime}\left(e_{2}, e_{2}\right)=1, h_{p}^{\prime}\left(e_{2}, e_{j}\right)=l\left(e_{j}\right)$, $j \in\{3, \ldots, s\}$, and $h_{p}^{\prime}\left(e_{i}, e_{j}\right)=\left\langle e_{i}, e_{j}\right\rangle$ for all $i, j \in\{3, \ldots, s\}$, is a lightlike product on $T_{p} M$ which extends to a $G$-homogeneous lightlike metric on $M$. Reciprocally, any $G$-homogeneous lightlike metric is homothetic to one of the previous form. Thus, there is a finite-dimensional family of $G$-homogeneous lightlike metrics on $M$, parametrized up to homothety, by the linear forms on $\mathbb{R}^{\mathrm{d} i m M-2}$.

4.4. Proof of Corollary 1.4. Here, we assume that $G$ is semisimple, noncompact, with finite center. The group $G$ acts transitively and nonproperly on a lightlike manifold $(M, h)$. Looking at a finite cover of $G$ if necessary, we assume that $G=$ $H_{1} \times \ldots \times H_{s}$, where each $H_{i}$ is a simple group with finite center. For $p \in M$ and every $i=1, \ldots, s$ we call $G_{p}^{i}$ the projection of the isotropy group $G_{p}$ on $H_{i}$, and $H_{p}^{i}$ the intersection $G_{p} \cap H_{i}$. Each $H_{p}^{i}$ is a normal subgroup of $G_{p}$. Since $G_{p}$ is noncompact, some $G_{p}^{i}$ has noncompact closure; for example $i=1$. Let us consider a sequence $\left(g_{k}\right)$ tending to infinity in $G_{p}$, such that the projection of $\left(g_{k}\right)$ on $H_{1}$ also tends to infinity. Performing a Cartan decomposition $\left(g_{k}\right)$ in $G$ and using (the proof of) Proposition 3.2, we get $X$ in a Cartan subalgebra of $\mathfrak{g}$ and $p^{\prime} \in M$ such that $W_{X}^{s}$ is isotropic at $p^{\prime}$. Now, because the projection of $\left(g_{k}\right)$ on $H_{1}$ tends to infinity and the Cartan decomposition in $G$ is obtained as a product of Cartan decompositions in the $H_{i}$ 's, we have that the projection $X_{1}$ of $X$ on $\mathfrak{h}_{1}$ is nontrivial. Moreover, as already observed, $W_{X_{1}, \mathfrak{h}_{1}}^{s}$ is isotropic at $p^{\prime}$. If $H_{1}$ is not locally isomorphic to $S L(2, \mathbb{R})$, we get that $W_{X_{1}, \mathfrak{h}_{1}}^{s}$ has dimension greater than 1 , and thus $H_{1} \cdot p^{\prime}$ is lightlike and carries a nonproper action of $H_{1}$. By the previous study, $H_{1}$ is isomorphic to $O(1, n), n \geq 3$, and $H_{1} \cdot p^{\prime}$ is homothetic to $C o^{n}$. We can then apply Proposition 4.5 to conclude.

We are left with the case where $H_{1}$ is a finite cover of $P S L(2, \mathbb{R})$ and $G_{p}^{1}$ does not have compact closure. We claim that the orbit $H_{1} . p$ cannot have dimension 3. Indeed, let \langle\rangle$_{p}$ be the pullback of $h_{p}$ in the Lie algebra $\mathfrak{h}_{1}$. Let $g \in G_{p}$ and $g_{j}$ be the projection of $g$ on $H_{j}$. Since $D_{p} g$ leaves $h_{p}$ invariant, we get that \langle\rangle$_{p}$ is $A d\left(g_{1}\right)$-invariant. But \langle\rangle$_{p}$ is either Riemannian or lightlike. In both cases, we saw in the proof of Proposition 2.1 that the subgroup $S \subset H_{1}$ such that $\operatorname{Ad}(S)$ preserves \langle\rangle$_{p}$ is compact, contradicting the fact that $G_{p}^{1}$ does not have compact closure.

If $H_{1} . p$ is of dimension 2 and Riemannian, $H_{p}^{1}$ is a maximal compact subgroup $K \subset H_{1}$. But since $H_{p}^{1}$ is normal in $G_{p}$, we get that $K$ is normal in $G_{p}^{1}$, which yields $G_{p}^{1}=K$ and a new contradiction. 
We conclude that $H_{1} . p$ is either 1-dimensional and lightlike, or 2-dimensional and lightlike. It follows from Proposition 2.1 that $H_{1} . p$ is homothetic to a cone $C o^{1}$ or $\mathrm{Co}^{2}$. We then get the conclusion thanks to Proposition 4.5.

\section{Proof of Theorem 1.5}

Here we assume that $G$ is simple with finite center and acts locally faithfully by isometries on a compact lightlike manifold $(M, h)$.

We first assume, by contradiction, that $G$ is not locally isomorphic to $\operatorname{PSL}(2, \mathbb{R})$. By compactness, every sequence of $M$ is nonescaping. It follows from the first point of Proposition 3.2 that for every $X$ in a Cartan subalgebra of $\mathfrak{g}, W_{X}^{s}$ is isotropic at every $p \in M$. Thus, using the last point of Lemma 4.1, and the conclusions of Corollary 1.4, we get that $G$ is locally isomorphic to $O(1, n)$, and any $G$-orbit is homothetic to $C o^{n}, n \geq 3$. Let us call $K$ a maximal compact subgroup of $G$, and let $K_{0}$ be the stabilizer in $K$ of a given point $p_{0} \in M$. As we said in the proof of Lemma 3.5. the compact group $K_{0}$ preserves a Riemannian metric on $M$. Since any Riemannian isometry can be linearized around any fixed point (via the exponential map), it is not difficult to prove that the set of $K_{0}$-fixed points is a closed submanifold of $M$ that we call $M_{0}$. We know explicitly the action of $K$ on $C o^{n}$, and observe that every orbit of $K$ is of Riemannian type. Let $S\left(\mathfrak{k} / \mathfrak{k}_{0}\right)$ denote the set of Euclidean scalar products on $\mathfrak{k} / \mathfrak{k}_{0}$. There is a continuous map $\mu: M_{0} \rightarrow S\left(\mathfrak{k} / \mathfrak{k}_{0}\right)$ defined in the following way: if $X$ and $Y$ are two vectors of $\mathfrak{k}$, and $\bar{X}$ and $\bar{Y}$ are their projections on $\mathfrak{k} / \mathfrak{k}_{0}$, then $\mu(p)(\bar{X}, \bar{Y})=h_{p}(X(p), Y(p))$. Now, any linear homothetic transformation of Minkowski space preserves $C o^{n}$, acts on it by a homothetic transformation for the lightlike metric, and commutes with any isometry of $C o^{n}$. As a consequence, on $G$. $p_{0}$, there is a 1-parameter flow of homotheties $h^{t}$ which transforms $h_{\mid G . p_{0}}$ into $e^{2 t} h_{\mid G . p_{0}}$ and commutes with the action of $K$ (in particular, it leaves $M_{0} \cap G$. $p_{0}$ invariant). It follows that $\mu\left(h^{t} \cdot p_{0}\right)=e^{2 t} \mu\left(p_{0}\right)$. Now, by compactness of $M_{0}$, there is a sequence $\left(t_{k}\right)$ tending to $+\infty$ such that $h^{t_{k}} \cdot p_{0}$ tends to $p_{\infty} \in M_{0}$. We should get by continuity of $\mu, \lim _{k \rightarrow+\infty} e^{2 t_{k}} \mu\left(p_{0}\right)=\mu\left(p_{\infty}\right)$, which yields the desired contradiction.

It remains to understand what happens if $G$ has finite center and is locally isomorphic to $P S L(2, \mathbb{R})$. Let us fix $X, Y, Z$ as a standard basis of $\mathfrak{g}:[Y, Z]=X$, $[X, Y]=2 Y$, and $[X, Z]=-2 Z$. It follows from Proposition 3.2 that $Y$ and $Z$ are isotropic at every $p \in M$. As a consequence, at any $p \in M$ a nontrivial linear combination of $Y$ and $Z$ has to vanish, so that all the orbits of $G$ have dimension at most 2 and are lightlike since stabilizers of points are noncompact. If there is a 2-dimensional orbit G.p. $p_{0}$, Proposition 2.1 ensures that it is homothetic to $\mathbb{R}^{2} \backslash\{0\}$ endowed with the metric $d \theta^{2}$ (namely $C o^{2}$ ). We get a contradiction exactly as above, using the action of a maximal compact group and the homothetic flow on $C o^{2}$ (here $\left.\mathfrak{k}_{0}=0\right)$.

We conclude that every $G$ orbit is 1-dimensional and lightlike. Since $G$ has finite center, these orbits are finite coverings of the circle, hence closed.

\section{Proof of Theorem 1.6}

Let us first summarize results on Lorentz dynamics in the following statement, fully proved in [9, but previously partially proved for instance in [1, 2, 4, 16]. 
Theorem 6.1. Let $G$ be a semisimple group with finite center, no compact factor and no local factor isomorphic to $S L(2, \mathbb{R})$, acting isometrically nonproperly on a Lorentz manifold $M$. Then, up to a finite cover, $G$ has a factor $G^{\prime}$ isomorphic to $O(1, n)$ or $O(2, n)$ and having some orbit homothetic to $d S_{n}$ or $A d S_{n}$.

Observe now that most developments along the article, in particular Proposition 3.2. do not explicitly involve the lightlike nature of the ambient metric and apply equally to the Lorentz case and to the general sub-Lorentz case. This allows one to find a nonproper $G$-orbit $O$, i.e. with a stabilizer algebra containing nilpotent elements (see the end of proof of Proposition 3.1). Let $x_{0} \in O$.

If the ambient metric at $x_{0}$ is Lorentz, then we just apply Theorem 6.1 to the Lorentz manifold $M^{\prime}$ which is the open subset of $M$ comprising the points where the metric is Lorentz.

If the metric at $x_{0}$ is of Riemannian or lightlike type, then we first observe that the $G$-action on $O$ is locally faithful. The proof in this mixed situation proceeds exactly as that of Lemma 3.5 in the (pure) lightlike case. By contradiction, if a factor $H$ of $G$ fixes $O$ pointwise, then its infinitesimal isotropy at $x_{0}$ is trivial (because the metric at $x_{0}$ is Riemannian or lightlike). Hence the action of any connected compact subgroup of $H$ is trivial, contradicting the fact that $G$ acts faithfully (see the proof of Lemma 3.5). Now, since the stabilizer algebra at $x_{0}$ contains nilpotent elements, and since the action on $O$ is locally faithful, we get that $O$ cannot be Riemannian, hence is lightlike. We then apply Theorem 1.3 to deduce that $O$ is homothetic to a lightcone $C o^{n}$ (up to a finite cover).

6.0.1. Some remaining questions. The results of $[9$ are stronger than the statement of Theorem 6.1] since they contain a detailed geometric description of the Lorentz manifold $M$ (a warped product structure...). This is the missing part of Theorem 1.3 in the lightlike nonhomogeneous case and Theorem 1.6 in the sub-Lorentz case. In particular, in this last sub-Lorentz situation it remains to see whether the manifold is or is not pure, i.e. if we can find examples of nonproper actions where a lightlike part and a Lorentz one coexist.

\section{ACKNOWLedGement}

We would like to thank the referee for her/his valuable remarks.

\section{REFERENCES}

[1] S. Adams: Orbit nonproper actions on Lorentz manifolds, Geom. Funct. Anal. 11 (2001), no. 2, 201-243. MR1837363 (2002f:37048)

[2] S. Adams: Dynamics of semisimple Lie groups on Lorentz manifolds, Geom. Ded. 105 (2004), 1-12. MR2057240 (2005d:37050)

[3] M. Akivis, V. Goldberg, On some methods of construction of invariant normalizations of lightlike hypersurfaces. Differential Geom. Appl. 12 (2000), no. 2, 121-143. MR.1758845 (2001f:53144)

[4] A. Arouche, M. Deffaf, A. Zeghib, On Lorentz dynamics: From group actions to warped products via homogeneous spaces. Trans. Amer. Math. Soc. 359 (2007), 1253-1263 MR2262849 (2008b:53091)

[5] E. Bekkara, C. Frances, A. Zeghib, On lightlike geometry: isometric actions, and rigidity aspects. C. R. Math. Acad. Sci. Paris 343 (2006), no. 5, 317-321. MR2253050 (2007e:53045)

[6] F. Bonsante, Flat spacetimes with compact hyperbolic Cauchy surfaces. J. Differential Geom. 69 (2005), 441-521. MR2170277 (2006h:53068)

[7] Y. Carrière, Flots riemanniens. Astérisque No. 116 (1984), 31-52. MR755161 (86m:58125a) 
[8] G. D'Ambra, M. Gromov, Lectures on transformation groups: geometry and dynamics. Surveys in differential geometry (Cambridge, MA, 1990), 19-111, Lehigh Univ., Bethlehem, PA, 199. MR:1144526 (93d:58117)

[9] M. Deffaf, K. Melnick, A. Zeghib, Actions of noncompact semisimple groups on Lorentz manifolds, Geom. Funct. Anal. 18 (2008), no. 2, 463-488. MR2421545 (2009g:57059)

[10] K. Duggal, A. Bejancu, Lightlike submanifolds of semi-Riemannian manifolds and applications. Mathematics and its Applications, 364. Kluwer Academic Publishers Group, Dordrecht, 1996. MR1383318(97e:53121)

[11] P Eberlein: Geometry of Nonpositively Curved Manifolds, University of Chicago Press, Chicago, 1996. MR1441541 (98h:53002)

[12] F. Friedlander, The wave equation on a curved space-time. Cambridge Monographs on Mathematical Physics, No. 2. Cambridge University Press, Cambridge-New York-Melbourne, 1975. MR0460898 (57:889)

[13] S. Hawking, G. Ellis, The large scale structure of space-time. Cambridge Monographs on Mathematical Physics, No. 1. Cambridge University Press, London-New York, 1973. MR0424186 (54:12154)

[14] A. Knapp, Lie groups beyond an introduction. Second edition. Progress in Mathematics, 140. Birkhäuser Boston, Inc., Boston, MA, 2002. MR1920389 (2003c:22001)

[15] T. Kobayashi, T. Yoshino, Compact Clifford-Klein form of symmetric spaces -revisited, Pure Appl. Math. Q. 1, (2005), no. 3, 591-663. MR2201328 (2007h:22013)

[16] N. Kowalsky, Noncompact simple automorphism groups of Lorentz manifolds. Ann. Math. 144 (1997), 611-640. MR 1426887 (98g:57059)

[17] D. Kupeli, Singular semi-Riemannian geometry. With the collaboration of Eduardo GarcaRo on Part III. Mathematics and its Applications, 366. Kluwer Academic Publishers Group, Dordrecht, 1996. MR.1392222 (97f:53105)

[18] T. Miernowski, Thesis, école normale supérieure de Lyon, 2005.

[19] P. Molino, Riemannian foliations. Progress in Mathematics, 73. Birkhuser Boston, Inc., Boston, MA, 1988. MR932463 (89b:53054)

[20] P. Chruściel, On rigidity of analytic black holes. Comm. Math. Phys. 189 (1997), 1-7. MR.1478527 (98j:83054)

[21] I. Robinson, A. Trautman, Integrable optical geometry, Lett. Math. Phys. 10 (1985), 179-182. MR815241 (87f:53070)

[22] K. Scannell, Flat conformal structures and the classification of de Sitter manifolds, Comm. Anal. Geom. 7 (1999), no. 2, 325-345. MR.1685590(2000a:53122)

[23] A. Zeghib, Geodesic foliations in Lorentz 3-manifolds. Comment. Math. Helv. 74 (1999), 1-21. MR:1677118 (99k:53049)

[24] A. Zeghib, Isometry groups and geodesic foliations of Lorentz manifolds. II. Geometry of analytic Lorentz manifolds with large isometry groups. Geom. Funct. Anal. 9 (1999), 823854. MR.1719610 (2001g:53060)

Department of Mathematics and Information, BP1523, ENSET of Oran, 31000 El M'naouar Oran, Algeria

E-mail address: esmaa.bekkara@gmail.com

Laboratoire de Mathématiques, Université Paris-Sud, 91405 Orsay Cedex, France

E-mail address: charles.frances@math.u-psud.fr

CNRS, UMPA, École Normale Supérieure de Lyon, 69364 Lyon Cexex 07, France

E-mail address: zeghib@umpa.ens-lyon.fr 